\title{
Guiding topological semimetals towards water oxidation in a Kagomé crystal lattice
}

\author{
GuangCun Shan ${ }^{1,2^{*}}$, and JiLiang Zhang ${ }^{3}$ \\ ${ }^{1}$ School of Instrument Science and Opto-electronic Engineering \& Institute of Quantum Sensing, Beihang University, Beijing 100083, China; \\ ${ }^{2}$ Max Planck Institute for Chemical Physics of Solids, Dresden D-01187, Germany; \\ ${ }^{3}$ Department of Energy \& Materials Engineering, Dongguk University, Seoul 100-715, Republic of Korea
}

Received July 23, 2019; accepted October 10, 2019; published online November 25, 2019

Citation: G. C. Shan, and J. L. Zhang, Guiding topological semimetals towards water oxidation in a Kagomé crystal lattice, Sci. China-Phys. Mech. Astron. 63, 237032 (2020), https://doi.org/10.1007/s11433-019-1456-0

Photocatalytic splitting of relatively plentiful water into $\mathrm{O}_{2}$ and $\mathrm{H}_{2}$ supplies a possible solution for storing solar energy to meet energy demands and environmental requirements [1]. The water oxidation half reaction to form dioxygen known as the oxygen evolution reaction (OER, $2 \mathrm{H}_{2} \mathrm{O} \rightarrow \mathrm{O}^{2}+4 \mathrm{H}^{+}+4 \mathrm{e}^{-}$) is attracting more attention because there are already known materials for efficient mediation of the reduction step $\left(4 \mathrm{H}^{+}\right.$ $+4 \mathrm{e}^{-} \rightarrow 2 \mathrm{H}_{2}$ ) [2]. Current research focuses on understanding the mechanisms of OER and development of new OER catalysts. The widely studied OER systems include platinum surface [1], transition metal oxides [2], transition metal complexes [3], 3d transition metal spinels and perovskites [4], and metal-organic framework (MOF) materials based on 3d transition metals [5]. In these materials, transition metals in the surface generally serve as active centers for the OER, while the surrounding atomic environments determine their performance and stability [6]. However, preparation of the surface has a large effect on reactivity (defects, kinks, low coordinate sites) therefore it is difficult to predict an OER material's properties by its bulk structure.

Now, writing in the Science Advances, Prof. Felser and her collaborators provide a strategy to explore a new kind of OER catalyst employing the topological surface states of topological semimetal, based on transition-metal Kagomé lattices as OER surfaces, which has a good metallic con-

*Corresponding author (email: gshan2-c@my.cityu.edu.hk; gcshan@buaa.edu.cn) ductivity [7]. Here it is worthwhile mentioning that Weyl semimetals (WSMs) that host exotic quasiparticles called Weyl fermions must break either spatial inversion or timereversal symmetry; and more importantly, $\mathrm{Co}_{3} \mathrm{Sn}_{2} \mathrm{~S}_{2}$ is the first magnetic WSM reported with broken time-reversalsymmetry [8], though a number of WSMs that break inversion symmetry have been identified.

As illustrated in Figure 1 [9], the non-zero electronic density of state (DOS) at the Fermi level indicative of the metallic conductivity and its valley shape suggest the structural stability of topological semimetals. Unlike the easily destroyable surface states derived by defects or doping, such topological surface states (TSSs) are robust against surface modifications and defects, while the partially filled $\mathrm{e}_{\mathrm{g}}$ orbital of the transition metal points to the $p$ orbital of the adsorbed hydroxide ions (Figure 2), which favours electron
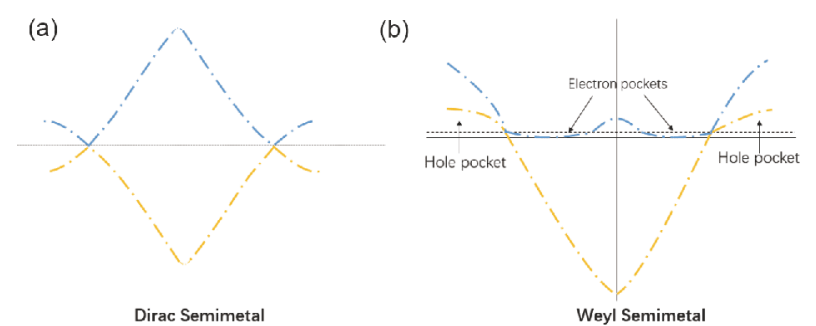

Figure 1 (Color online) Schematic illustrations of band structures for (a) Dirac Semimetal, (b) Weyl Semimetal. 
(a)

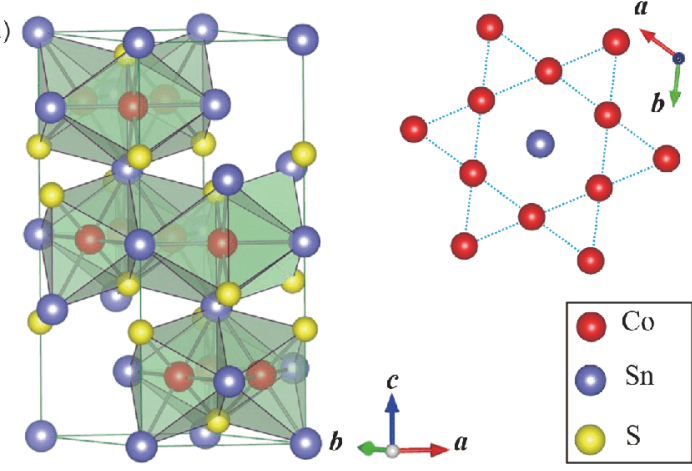

(b)

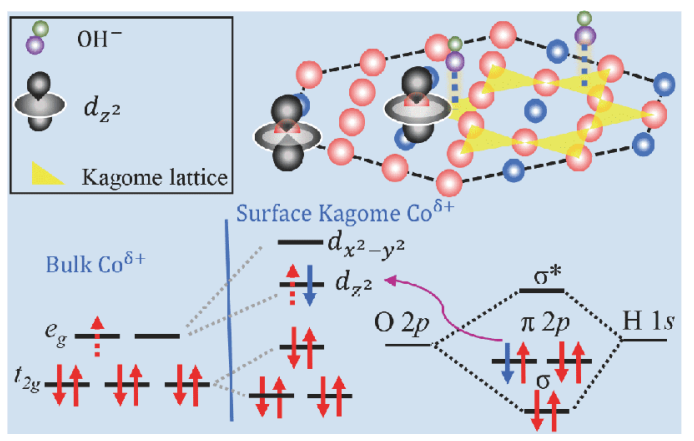

Figure 2 (Color online) (a) Crystal lattice structure of $\mathrm{Co}_{3} \mathrm{Sn}_{2} \mathrm{~S}_{2}$ with Kagomé lattice; (b) schematic illustration of electrochemical catalytic mechanism in $\mathrm{Co}_{3} \mathrm{Sn}_{2} \mathrm{~S}_{2}$ topological semimetal. Reprinted with permission from Science Advances, aaw9867 (2019). (C) Copyright 2019 by American Association for the Advancement of Science.

transfer and strengths the bonds between the adsorbate and catalytic sites. Despite the smaller surface area, the bulk single crystal of studied compound $\mathrm{Co}_{3} \mathrm{Sn}_{2} \mathrm{~S}_{2}$, already shows outstanding OER catalytic performance compared with Cobased nanostructured catalysts, including fast kinetics and low overpotential [7]. Generally a more stable surface state suggests less activity in catalysts [10]. The filling of $e_{g}$ orbital corresponds to a higher energy state for high activity, while the topological character of Kagomé lattice stabilizes this configuration. Obviously, the cooperation of electronic and topological factors in that work supplies a promising way to tune topological nontrivial surface states for the rational design of high-performance OER catalysts. In addition, compared to non-magnetic topological semimetal, for magnetic semimetal $\mathrm{Co}_{3} \mathrm{Sn}_{2} \mathrm{~S}_{2}$, the magnetism is necessary to achieve high performance in OER.

Despite the success of this new strategy on topological WSM $\mathrm{Co}_{3} \mathrm{Sn}_{2} \mathrm{~S}_{2}$, some issues have to be overcome to develop new novel OER catalysts. Most topological semimetals are made as bulk single crystals in order to measure their physical properties. As a result, more efforts are required to produce the thin film or nanostructure forms of these magnetic semimetal materials as exposed surfaces for high OER performance. Many topological semimetals are not based on transition metals and thus not suitable for OER catalysts $[11,12]$, limiting the application of the proposed strategy.

However, the idea presented in Felser and her collaborators' work can be extended to other systems, i.e., introducing unpaired electrons to improve OER activity in a Kagomé lattice, or stabilizing active OER catalysts by structural changes like Jahn-Teller distortions. Besides, the Kagomé lattice of WSM $\mathrm{Co}_{3} \mathrm{Sn}_{2} \mathrm{~S}_{2}$ would be advantageous for OER, which is quasi-layered lattice structure and consists of transitional metal Co serving as the main active site for OER. It is further demonstrated by the scanning tunneling spectroscopy (STS) measurements of Morali et al. [13] that this quasilayered structure of $\mathrm{Co}_{3} \mathrm{Sn}_{2} \mathrm{~S}_{2}$ facilitates exposure of its crystal faces consisting of Kagomé lattice, which could accommodate the active sites of Co for OER. The OER catalytic process, especially the adsorption and charge transfer of the reaction intermediates, can be effectively modulated by tuning some of the physical properties of the Kagomé lattice layer, such as magnetism, conductivity and mobility.

In recent years, topological semimetal has been one of the hottest topics in condensed matter physics [8,9,13-17], and particularly, WSMs materials hosting exotic quasiparticles of Weyl fermions must break either spatial inversion or timereversal symmetry. Interestingly, using angle-resolved photoemission spectroscopy, together with Felser and Parkin from Germany, Chen and his collaborators [17] have further visualized the electronic structure of the ferromagnetic crystal $\mathrm{Co}_{3} \mathrm{Sn}_{2} \mathrm{~S}_{2}$ and discovered its characteristic surface Fermi-arcs and linear bulk band dispersions across the Weyl points. These new interesting results establish $\mathrm{Co}_{3} \mathrm{Sn}_{2} \mathrm{~S}_{2}$ as a magnetic Weyl semimetal, which might serve as a platform not only for realizing fascinating phenomena such as chiral magnetic effects, unusual large anomalous Hall effect and quantum anomalous Hall effect, but also for making more efficient OER catalysts for water splitting.

This work was supported by the National Key R\&D Program of China (Grant No. 2016YFE0204200). We thank Dr. GuoWei Li at Max-Planck Institute and Dr. YuLin Chen at Oxford University for their insightful and interesting discussions relating to this work.

1 D. Strmcnik, M. Uchimura, C. Wang, R. Subbaraman, N. Danilovic, D. van der Vliet, A. P. Paulikas, V. R. Stamenkovic, and N. M. Markovic, Nat. Chem. 5, 300 (2013).

2 S. Trasatti, Electrochim. Acta 29, 1503 (1984).

3 R. Matheu, P. Garrido-Barros, M. Gil-Sepulcre, M. Z. Ertem, X. Sala, C. Gimbert-Suriñach, and A. Llobet, Nat. Rev. Chem. 3, 331 (2019).

4 I. Godwin, A. Rovetta, M. Lyons, and J. Coleman, Curr. Opin. Electrochem. 7, 31 (2018).

5 B. Nepal, and S. Das, Angew. Chem. Int. Ed. 52, 7224 (2013).

6 V. Tripkovic, H. A. Hansen, and T. Vegge, ACS Catal. 7, 8558 (2017).

7 G. Li, Q. Xu, W. Shi, C. Fu, L. Jiao, M. E. Kamminga, M. Yu, H. Tüysüz, N. Kumar, V. Süß, R. Saha, A. K. Srivastava, S. Wirth, G. Auffermann, J. Gooth, S. Parkin, Y. Sun, E. Liu, and C. Felser, Sci. Adv. 5, eaaw9867 (2019).

8 E. Liu, Y. Sun, N. Kumar, L. Muechler, A. Sun, L. Jiao, S. Y. Yang, D. Liu, A. Liang, Q. Xu, J. Kroder, V. Süß, H. Borrmann, C. Shekhar, Z. Wang, C. Xi, W. Wang, W. Schnelle, S. Wirth, Y. Chen, S. T. B. 
Goennenwein, and C. Felser, Nat. Phys. 14, 1125 (2018), arXiv: 1712.06722.

9 A. A. Burkov, Nat. Mater. 15, 1145 (2016), arXiv: 1610.07866.

10 S. H. Chang, J. G. Connell, N. Danilovic, R. Subbaraman, K. C. Chang, V. R. Stamenkovic, and N. M. Markovic, Faraday Discuss. 176, 125 (2014).

11 R. Ma, G. Lin, Y. Zhou, Q. Liu, T. Zhang, G. Shan, M. Yang, and J. Wang, npj Comput. Mater. 5, 78 (2019).

12 A. Bernevig, H. Weng, Z. Fang, and X. Dai, J. Phys. Soc. Jpn. 87, 041001 (2018).

13 N. Morali, R. Batabyal, P. K. Nag, E. Liu, Q. Xu, Y. Sun, B. Yan, C. Felser, N. Avraham, and H. Beidenkopf, Science 365, 1286 (2019).
14 H. Weng, C. Fang, Z. Fang, B. A. Bernevig, and X. Dai, Phys. Rev. X 5, 011029 (2015), arXiv: 1501.00060.

15 Y. P. Li, Z. Wang, P. S. Li, X. J. Yang, Z. X. Shen, F. Sheng, X. D. Li, Y. Lu, Y. Zheng, and Z. A. Xu, Front. Phys. 12, 127205 (2017), arXiv: 1612.04031.

16 W. Gao, N. Hao, F. W. Zheng, W. Ning, M. Wu, X. Zhu, G. Zheng, J. Zhang, J. Lu, H. Zhang, C. Xi, J. Yang, H. Du, P. Zhang, Y. Zhang, and M. Tian, Phys. Rev. Lett. 118, 256601 (2017), arXiv: 1609.02626.

17 D. F. Liu, A. J. Liang, E. K. Liu, Q. N. Xu, Y. W. Li, C. Chen, D. Pei, W. J. Shi, S. K. Mo, P. Dudin, T. Kim, C. Cacho, G. Li, Y. Sun, L. X. Yang, Z. K. Liu, S. S. P. Parkin, C. Felser, and Y. L. Chen, Science 365, 1282 (2019). 